\title{
Cosmological Applications of the Gaussian Kinematic Formula
}

\author{
Yabebal T. Fantaye ${ }^{1}$ and Domenico Marinucci ${ }^{1} \dagger$ \\ ${ }^{1}$ Dipartimento di Matematica, Universit di Roma"Tor Vergata, \\ Via della Ricerca Scientifica 1, I-00133 Roma, Italy \\ email: fantaye@mat.uniroma2.it, marinucc@axp.mat.uniroma2.it
}

\begin{abstract}
The Gaussian Kinematic Formula (GKF, see Adler and Taylor $(2007,2011)$ ) is an extremely powerful tool allowing for explicit analytic predictions of expected values of Minkowski functionals under realistic experimental conditions for cosmological data collections. In this paper, we implement Minkowski functionals on multipoles and needlet components of CMB fields, thus allowing a better control of cosmic variance and extraction of information on both harmonic and real domains; we then exploit the GKF to provide their expected values on spherical maps, in the presence of arbitrary sky masks, and under nonGaussian circumstances.
\end{abstract}

Keywords. methods: analytical,methods: data analysis,methods: numerical ,methods: statistical, techniques: image processing, cosmology: cosmic microwave background

\section{Introduction}

The expected values of the Minkowski functionals in the planar case and under Gaussianity is analytically known to the literature since the work of Adler in the early 80's Adler 1981, see also Tomita 1986. A major advancement of this field in the last dacade, however, was brought by the discovery of the Gaussian Kinematic Formula (GKF) by Adler and Taylor $(2003,2007,2009)$. GKF allows a simple computation of the expected values for Lipschitz-Killing curvatures (LKCs) (which are equivalent to Minkowski functionals up to a constant factor) under an impressive variety of extremely different circumstances, covering arbitrary manifolds with and without masked regions and a broad class of nonGaussian models.

One of our purposes in this paper is to exploit these recent results to develop a number of analytic predictions on functionals tailored to test nonGaussianities and asymmetries on CMB data. Due to page constraints, here we will show only results for Gaussian fields convolved with a realistic mask in needlet domain. For detail analysis and explicit analytical expressions of the expected values of LKCs for Gaussian and nonGaussian cases on multipole and needlet domains and including an arbitrary sky cuts, please refer to our paper Fantaye et. al. 2014.

\section{Gaussian Kinematic Formula (GKF)}

The GKF is about expected values of Lipschitz-Killing curvatures for excursion regions. The great power of the GKF is that it allows for a full decoupling of the expected values of LKCs of an excursion set $\mathbb{E} \mathcal{L}_{i}\left(A_{u}(g(T), M)\right)$ into components which are completely independent: the LKCs of the original manifold $\mathcal{L}_{i+l}(M)$, the Gaussian Minkowski Functionals which depends only threshold value $\mathcal{M}(u)$, and the covariance structure $\lambda$ of the field. The independence of these components to each other means an enormous

$\dagger$ Research supported by ERC Grant 277742 Pascal. 
computational advantages to derive analytical results under a variety of circumstances, including masked regions as we shall see below. Before giving the expression of the GKF, let us define the important components in turn.

Exursion sets: $\left.A_{u}(f(x), M)\right)$

On the sphere, the excursion sets $A_{u}(f)$ of a given (possibly random) function $f$ are defined as

$$
A_{u}(f):=\left\{x \in S^{2}: f(x) \geqslant u\right\} .
$$

Of course, in the limit where we take $u=-\infty$, we have that $A_{u}(f)=S^{2}$.

Lipschitz-Killing Curvatures (LKCs): $\mathbb{E} \mathcal{L}_{i}\left(A_{u}(f(x), M)\right)$

The LKCs for the region $A$ with dimension $\operatorname{dim}(A)=n$, are defined as the coefficients of a Taylor expansion of a Tube of radius $r$ around $A$.

$$
\operatorname{Vol}[\operatorname{Tube}(A, r)]=\sum_{k=0}^{n} \mathcal{L}_{n-k}(A) \omega_{k} r^{k} .
$$

where $\omega_{k}$ are the volume of a unit ball in $\mathcal{R}^{k}$. LKCs depend on the Riemannian metric, and are a measure of the k-dimensional size of the Riemannian manifold $M$. For instance, let $A$ be the unit square on the plane; by elementary geometry, the volume of the Tube is then given by

$$
\mathcal{L}_{2}(A)+2 \mathcal{L}_{1}(A) r+\mathcal{L}_{0}(A) \pi r^{2}=1+2 \cdot 2 \cdot r+\pi r^{2},
$$

the LKCs $\mathcal{L}_{0}, \mathcal{L}_{1}, \mathcal{L}_{2}$ correspond to Euler-Poincaré characteristic, half the boundary length and area, respectively.

\section{Gaussian Minkowski Functionals (GMFs): $\mathcal{M}_{k}(U)$}

The Gaussian Minkowski Functionals (GMFs) $\mathcal{M}_{k}(U)$ are defined as the Taylor coefficients in the expansion of the Tube probabilities $\operatorname{Pr}\{Z \in T u b e(U, r)\}$, the probability that a zero-mean standard Gaussian variable belongs to Tube $(U, r)$; for instance, for $U=[u, \infty)$. The GMFs dependence only on the excursion threshold $u$ and can be easily computed using the following expression

$$
\mathcal{M}_{j}^{\gamma_{k}}([u, \infty))=(2 \pi)^{-1 / 2} H_{j-1}(u) e^{-u^{2} / 2} .
$$

where $H_{j}$ is the Hermite polynomials: $H_{0}(u)=1, H_{1}(u)=2 u, H_{2}(u)=4 u^{2}-1, H_{3}(u)=$ $8 u^{3}-12 u$.

\section{Metric scaling coefficients: $\lambda^{1 / 2}$}

The metric scaling coefficient $\lambda$ represents the covariance structure of the manifold we are working with, and are simply given by the second derivative of the covariance function at the origin. On a sphere the scaling $\lambda$ required to go to a needlet domain is given by:

$$
\lambda_{j}=\sqrt{\frac{\sum_{\ell} b^{2}\left(\frac{\ell}{2^{j}}\right) C_{\ell} \frac{2 \ell+1}{4 \pi} \frac{\ell(\ell+1)}{2}}{\sum_{\ell} b^{2}\left(\frac{\ell}{2^{j}}\right) C_{\ell} \frac{2 \ell+1}{4 \pi}}},
$$

where $b($.$) is the needlet weight function, j$ is the needlet frequency parameter, and $B>1$ is some fixed bandwidth. 
Under these circumstances, the Gaussian kinematic formula takes the form

$$
\lambda^{i / 2} \mathbb{E} \mathcal{L}_{i}\left(A_{u}(g(T), M)\right)=\sum_{k=0}^{\operatorname{dim}(M)-i} \lambda^{(i+k) / 2} \mathcal{L}_{i+k}(M) \mathcal{M}_{k}\left(g^{-1}[u, \infty)\right) ;
$$

\section{LKCs in the presence of sky cuts}

Assume we observe only $M:=S^{2} \backslash G$. Recall that $\mathcal{L}_{0}(M), \mathcal{L}_{1}(M) \mathcal{L}_{2}(M)$ are the LKCs of the unmasked region. Then the expected values of LKCs in the presence of mask $G$ are:

the Euler characteristic is given by

$$
\mathbb{E} \mathcal{L}_{0}\left(A_{u}(f(x), M)\right)=\{1-\Phi(u)\} \mathcal{L}_{0}(M)+\frac{1}{4} \lambda_{s} e^{-u^{2} / 2} \mathcal{L}_{1}(M)+\lambda_{s}^{2} \frac{u e^{-u^{2} / 2}}{\sqrt{(2 \pi)^{3}}} \mathcal{L}_{2}(M)
$$

half the boundary length

$$
\mathbb{E} \mathcal{L}_{1}\left(A_{u}(f(x), M)\right)=2\{1-\Phi(u)\} \mathcal{L}_{1}(M)+\frac{1}{4} \lambda_{s} e^{-u^{2} / 2} \mathcal{L}_{2}(M)
$$

the area

$$
\mathbb{E} \mathcal{L}_{2}\left(A_{u}\left(f(x), S^{2}\right)\right)=(1-\Phi(u)) \mathcal{L}_{2}\left(S^{2}\right) .
$$

For an arbitrary mask $G$ what is unknown in the above equations are the input LKCs $\mathcal{L}_{i}\left(S^{2} \backslash G\right)$. A very simple solution to derive these parameters can be provided by exploiting one more time Gaussian Kinematic Formula, following an idea discussed in Adler \& Taylor 2011, chapter 5.4. The strategy is as follows:

(a) Fix a simple power spectrum $C_{\ell}$, for instance with $L_{\max }=10$, and generate Gaussian maps out of it

(b) Fix a limited number of threshold values $u$ and perform a Monte Carlo evaluation of the LKCs evaluated on the excursion set of the fields generated according to (a) and with the mask $\mathrm{G}$ applied

(c) Use least square regression to estimate $\mathcal{L}_{i}\left(S^{2} \backslash G\right), i=0,1,2$

(d) Use the estimates obtained in point 3 as an input for equation (2.1) for any arbitrary power spectrum (for instance, multipole or needlet components on realizations of a $\Lambda C D M$ model, under Gaussian and nonGaussian circumstances).

\section{Numerical results}

To compare analytical results of LKCs with Monte Carlo simulations, we generated 100 realizations of an input power spectrum using the HEALpix Górski et. al. 2005 package. A root mean square normalized Gaussian needlet maps, which we used for all our analysis, are obtained by first generating spherical harmonic coefficients from the input power spectrum through the HEALpix create_alm routine up to a maximum multipole of $\ell_{\max }=2000$; and then by convolving these coefficients with the needlet filter as

$$
\beta_{j}(x)=\frac{1}{\sigma_{j}} \sum_{\ell=B^{j-1}}^{B^{j+1}} \sum_{m} b^{2}\left(\frac{\ell}{B^{j}}\right) a_{\ell m} Y_{\ell m}(x),
$$

where $\sigma_{j}^{2}=\frac{1}{4 \pi} \sum_{\ell} b^{4}\left(\frac{\ell}{B^{j}}\right)(2 \ell+1) C_{\ell}$ is the variance. We then applied a realistic mask on these maps. The mask used is shown in the left panel of Fig. (1). 

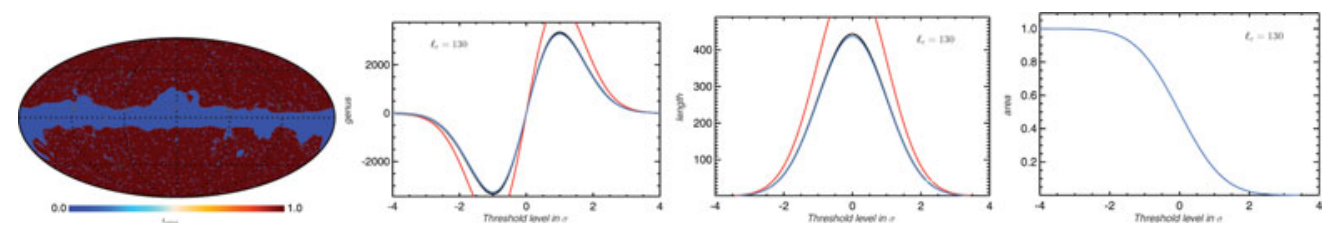

Figure 1. Masked LKCs on needlet space for a Gaussian field. Right panel shows the mask used in the analysis. The other three panels are for the Euler-Poincaré characteristic (genus), boundary length and area functionals. Analytical results are shown in red (assuming full sky) and blue (for mask corrected). Simulations are shown in black (mean of the simulations) and grey shades, which are 68,95 and $99 \%$ percentiles estimated from 100 simulations. The needlet parameters used are $B=1.5$ and $j=12$, which corresponds to multipole range $\ell=[87,195]$.

From these normalized and masked needlet maps we computed the three Minkowski Functionals, which as argued earlier are equivalent to the LKCs up to constant factors. This implementation is achieved exploiting the algorithms described in Eriksen et. al. 2004. The results we will show here are for a needlet frequency parameter $j=12$ and the band width $B=1.5$; this particular needlet map has a compact support for multipole ranges $\ell=[87,195]$. More details can be found in Fantaye et. al. 2014 .

To obtain the analytical results corresponding to our simulations, we first computed the mask dependent input LKCs, $\mathcal{L}\rangle\left(S^{2} / G\right)$, using a single multipole sumulation at multipole $\ell=15$ and following the procedures given in Section 3. We then substituted back these values to Eqns. $(3.1,3.2,3.3)$. The comparison of the analytical and simulation results for Euler-Poincaré characteristic, boundary length, and area are shown in Fig. (1).

\section{Summary and Conclusion}

The Gaussian Kinematic Formula allows to evaluate exact expected values for LipschitzKilling curvatures (Minkowski functionals) in a number of circumstances of applied interest, covering in particular full-sky experiments (accounting for the geometry of the sphere), nonlinear statistics and masked data.

We found an excellent agreement in all the cases that we investigated; more precisely, the analytical estimates are always well within the $68 \%$ Confidence Interval (CL) estimated from simulations, and as shown in the figures they are for practical purposes indistinguishable from the theoretical predictions even with a relatively low number of Monte Carlo simulations.

\section{References}

Planck Collaboration ArXiv e-prints: 1303.5083

Yabebal Fantaye, Frode Hansen, Davide Maino, Domenico Marinucci ArXiv e-prints: 1406.5420 R. J. Adler, John Wiley ES Sons Inc, ISBN: 0471278440 The Geometry of Random Fields 1981 J. E. Taylor \& R. J. Adler, The Annals of Probability 31, 533, 2003

Adler, Ewing, \& Taylor, Statistical Science 24, 1, 1009

Adler \& Taylor, Springer Berlin Heidelberg, ISBN: 978-3-642-19579-2 Topological Complexity of Smooth Random Functions 2007

Adler \& Taylor Springer New York, ISBN: 9780387481128 Random Fields and Geometry 2007

H. Tomita, Progress of Theoretical Physics 76, 952, 1986

Eriksen, Hansen, Banday, Gorski, and Lilje, ApJ 605,14, 2004 